\title{
Analysis of Radiological Terrorism on Metropolitan Area
}

\author{
Hosik Yoo (Main author) \\ KINAC 1534 Yuseong-daero, Daejeon, 305-348, Korea \\ Tel: 82-42-860-9782 E-mail: hsyoo@kinac.re.kr \\ Jeong-ho Lee (Corresponding author) \\ KINAC 1534 Yuseong-daero, Daejeon, 305-348, Korea \\ Tel: 82-42-860-9783Ｅ-mail: friend25kr@kinac.re.kr \\ Sung-woo Kwak (Corresponding author) \\ KINAC 1534 Yuseong-daero, Daejeon, 305-348, Korea \\ Tel: 82-42-860-9810Ｅ-mail: swkwak@kinac.re.kr
}

Received: September 14, $2011 \quad$ Accepted: September 29, $2011 \quad$ Published: December 31, 2011

doi:10.5539/eer.v1n1p24 URL: http://dx.doi.org/10.5539/eer.v1n1p24

The research is financed by Ministry of Education, Science and Technology in Korea.

\begin{abstract}
Possible terrorist attacks on metropolitan areas using radioactive materials have become the subject of lengthy research. This paper discusses an analysis that was conducted through a series of processes: selection of target areas, radioactive materials that may be used, scenario generation, analysis of risk by experts, and radiological consequences. A total of 336 different cases for evaluation were prepared using qualitative judgments; and a risk analysis was conducted to predict the consequence of a successful attack. A HOTSPOT code (which is widely used for initial assessments for emergency response planning) was adopted to evaluate radiological consequences from developed scenarios. We selected the target areas based on data such as: population, number of places where radioactive materials are stored and used, and quantity of radioactive materials. Seven different kinds of radioactive material and two kinds of explosives were considered in building the database. The results suggest that the dispersion distance from an explosion of radioactive material is dependent upon wind currents and the types of detrimental radioactive material utilized. Information from the database established by using radiological scenarios discussed in this study would be useful for countering a radiological attack at the early stag--from the perspective of physical protection.
\end{abstract}

Keywords: Radiological sabotage, Terrorism, Risk analysis, Metropolitan areas

\section{Introduction}

The possibility of an attack using a radioactive material was raised after the September 11, 2001 terrorist attacks in the U.S.A. An attack using a radiological dispersal device (RDD) is very attractive to terrorists because it can cause widespread damage to public health as well as leave long lasting psychological effects. The consequence of an attack would be more severe in a metropolitan area than in a remote area because of population density. The economic impact is also significant. Procuring radioactive materials for making a RDD would not difficult because of the increase use of radioactive materials and construction of storage facilities for their containment. Currently security for radioactive materials used in industrial and medical applications is poor compared with that of radioactive materials - fresh nuclear fuel and spent fuel - used in a nuclear power plant. The consequence of a RDD (dirty bomb) attack varies according to the type of radioactive material used. The typical indicator used to represent the consequences of an attack lists the level of radiation exposure to a human. It is determined mainly by the kinds of radioactive materials used. Another factor consider (from the perspective of physical protection) was how large an area could be contaminated. This information can give a clue in order to decide the 
extent of the evacuation area. The size of the contaminated area may be dependent upon the intensity of winds and the quantity of the explosives used. With the increasing potential for a radiological attack, many studies on the effects of an explosion of a RDD in a metropolitan area have been performed. Durante and Manti calculated the health effects caused by an explosive releasing one kg of plutonium detonated in the atmosphere over the city New York using the HOTSPOT computer codes. Kelly studied the influence of cesium, cobalt, and americium on human health. (There also have been other studies on the consequence of radiological terrorism in a city.)

Decision makers who are responsible for emergency situations can act accordingly to reduce casualties, based on the information that they can obtain during the initial stages of an attack. The prime concern for emergency response planners would be to obtain information on what kinds of radioactive material were used, how large the contaminated area is and how many people were exposed to lethal radiation. Several countries are developing tools to support and guide decision makers. The most important factor to be considered in developing evaluation tools is the time needed to calculate the damages caused by a RDD attack. Damages can be drastically reduced if evaluation results of the attack are provided quickly. Although the information during an emergency could be collected in short time, the information could be useless or inaccurate, making the situation worse. A trade-off between evaluation speed and accuracy should be considered. This paper suggests how to evaluate essential information quickly and with reasonable accuracy.

The best way to decrease the time needed to obtain these results would be to establish a database that includes virtual damages obtained from many possible scenarios. A scenario should include factors affecting the consequences of the attack such as: types of radioactive materials, direction and intensity of winds, and power of the explosive used. The consequences resulting from an attack may be easily available by just clicking a button to database and retrieving all possible case scenarios. Different databases should be established according to the region since attack scenarios may vary. This paper discussed the risk analysis of a RDD attack on a metropolitan area and presents a database constructed by the results obtained from this analysis.

\section{Establishment of Scenarios}

The establishment of plausible attack scenarios is a key element that will give credibility to any study. An attack scenario is comprised of various parameters, and its major components will be different depending on the purpose of the analysis. The scenario in this study calculates the radiological effects on humans and the environment. The parameters used in the study were based on: the type and quantity radioactive material to be used, meteorological data, and types of explosives and recommendations of based on reference material. The parameters used in scenarios are summarized in Table 1.

\subsection{Radioactive material}

There are many types of radioactive material that are used, stored and produced for academic, medical and industrial applications. The amount of this material is increasing steadily as their applications expand. In nuclear power plants and research facilities for nuclear energy, large amounts of harmful radioactive material are utilized, but it is so tightly protected that obtaining it illegally is extremely difficult. Radioactive materials are also being used in medical diagnosis, sterilization of medical equipment and for research purposes. The protection system in places where these materials are used is poor when compared with that of a nuclear power plant. We collected information about radioactive materials that are stored and used in metropolitan areas; and selected seven materials that are most frequently used and can be a source for a RDD.

\subsection{Explosive}

The radiological consequences of an attack using a RDD can vary with the amount of explosives used. Terrorists could use two different types of explosives depending on their purpose and target. The explosives could be hand carried loaded into a car. The DHS (Department of Homeland Security) in the U.S published a guide table on explosive attacks. The table lists the consequences if explosives are used, according to their amount. Based on this guide table, two quantities of TNT (50 lbs hand carried and 500 loaded in a car) were selected for analysis in this study.

\subsection{Meteorological condition}

One critical parameter in determining the size of the area that could be contaminated by radiological substances is the metrological condition at the time of an explosion. Meteorological factors include: direction and intensity of winds and stability of the atmosphere. It is not an easy task to determine the direction and intensity of winds. Analyzing their effects on the consequences of a RDD attack is difficult because they are so changeable. In order to get credible data, we surveyed climate data for the last 25 years from the meteorological office and performed a statistical treatment. Through this process, direction and intensity of winds as well as atmosphere stability were 
determined.

\subsection{Scenarios}

The purpose of a RDD attack is to inflict severe casualties. Places that are always crowded such as stations, hotels and markets have a higher probability of being targeted. A government building could be an attractive target for terrorist due to its symbolic status, as well as for the potential loss life and the long term economic damage. In this study, we performed an analysis on a possible RDD attack on a city hall located in a metropolitan area. There are many surrounding business buildings, markets and historical palaces where a great many people may visit daily. Such a location could prove an attractive target for terrorists. The radioactive materials we selected as source materials are categorized in three groups for analysis.

1). Radioactive Isotope (RI) material with low and medium radioactivity: Use of RI such as Ni-63, Ir-192, Cs-137. These materials could be stolen from a hospital or industrial facility.

2). RI material with high radioactivity: Theft of RI material such as Am-241 and Co-60 from an industrial facility.

3). Nuclear material: Theft of Pu-239 or LEU (less than 5\% U235) from a nuclear power reactor or nuclear fuel manufacturing plant.

RI materials could be obtained from a radiotherapy device in a hospital or from an irradiator in a nuclear facility. They can be transported quite easily to a target area since there are many hospitals and nuclear facilities near metropolitan areas. Compared to RI materials, theft of nuclear materials is difficult because of the strict security systems installed at nuclear power reactors and nuclear fuel manufacturing plants. Also transportation of nuclear materials to a target area is not an easy task since most of nuclear power plants are located in remote areas. Once radioactive materials are stolen, they would be transported to some place where a RDD could be constructed and then transported again to the target area. A scenario for a RDD attack is comprised of characteristics and attributes such as: motivation and capabilities of an adversary, as well as the types and amount of radioactive material and explosives used. However, we do not consider other parameters--geographical information, terrain, building type, the deposited radioactive materials--except for those that directly affect consequences. The objective of this study is to acquire information on the consequences of RDD attack for establishing a database. The parameters that would influence the radiological consequences include: the radioactive materials, the amount of explosives used and meteorological conditions (direction and intensity of wind, stability of atmosphere). Seven types of radioactive materials, two different types of explosives, and four types of meteorological conditions were considered. A total of 336 possible attack scenarios were examined.

\section{Analysis of Consequence}

There are several indexes used to help calculate the potential damage that could be caused by a RDD attack in terms of its economical, social and radiological impact. The economical and social damage cannot be easily calculated quantitatively and was beyond objective of this study. On the other hand, radiological damages can be reasonably estimated if reliable information is given. Some types of the radiological consequences can be considered; but the level of radioactivity and the contaminated area are the prime concerns to those who are responsible for the evacuation of the people who live near ground zero. In this study, we calculated the radiological consequences of 336 cases by using the HOPSPOT code and analyzed the results in terms of the types of radioactive materials used and other conditions. HOTSPOT evaluates a downwind assessment following the release of radioactive materials (continuous or puff release, explosive release, fuel fire or an area contamination event). Of these release models, explosive release was chosen in this paper. The HOTSPOT dose values are due solely to the inhalation of released material during passage of the plume. Analytical results of the HOTSPOT were described in terms of the TEDE (Total Effective Dose Equivalent) and contaminated area. Alpha-emissions must be more than several curie $(\mathrm{Ci})$ in intensity and gamma-emissions several hundred $\mathrm{Ci}$ in strength in order for radioactive materials to be effective as a dirty bomb. Thus, in calculation, the intensities of alpha-ray and gamma-ray sources (Am-241, Pu-239) are one $\mathrm{Ci}$ and $100 \mathrm{Ci}$, respectively, as shown in Table 1. LEU of $1,000 \mathrm{~kg}$ is used because it is less effective than gamma-ray source for the goal of a dirty bomb.

It was revealed that Am-241 was the most dangerous source because its toxicity in small quantities $(1 \mathrm{Ci})$ compared with other sources (Co-60, Cs-137: $100 \mathrm{Ci}$ ). The TEDE value from an explosion of Am-241 is higher than that of Pu-239 of the same quantity (see in Fig. 1). The results shown in Fig. 1 were obtained by an evaluation of a scenario in which radioactive materials were placed with $500 \mathrm{lbs}$ of explosives in a car. In the scenario, the car was exploded in a densely populated area, and the wind intensity at the site was $1.9 \mathrm{~m} / \mathrm{sec}$. Fig. 2 shows the TEDE value with distance under the various radioactive materials. There was no significant difference in the TEDE among four radioactive materials studied and their TEDE values are not high enough 
cause fatalities. The total values of the TEDE after an explosion of radioactive materials, such as Am-241, Pu-239 and Co-60 are higher than those of other materials (as can be seen in Table 2). It was meaningful work to compare the results of the TEDE value with the variations in the quantity of explosives and the intensity of winds. Fig. 3 shows the results of the calculations on the TEDE in four different conditions when Am-241 was used as a source material. It can be seen from Fig. 3 that the TEDE values decrease with an increase in the quantity of explosives and the intensity of winds. If the same amount of radioactive materials was detonated by a more powerful explosive, the radioactive particles would disperse over a wider area. A low value of the TEDE would be measured at the same distance compared to the case of an explosion by a less powerful explosive. The effect of the winds on radioactive particles is proportional to the quantity of explosives used. Radioactive particles diffuse more rapidly if the wind blows faster, which lowers the value of the TEDE at a certain distance.

\section{Discussion}

A radiological attack could cause severe damage to the population and the environment. A quick response is required to reduce damage. This can be accomplished by predicting the consequences that may result from an explosion of radioactive material. Many important factors should be considered when evaluating the consequences, but it would take long time to obtain the necessary information.

In an emergency, fast and accurate estimation of radiological consequence would be essential to minimize damage. However, if the calculation time is too long, the evaluation results would not contribute to establishing an effective emergency response plan. Thus, consideration might have to be given to a trade-off between calculation speed and accuracy. To address this problem, this paper suggests building a database where information requiring complex and time consuming calculations are already collected and analyzed. This study was performed in order to obtain data on the consequences of a radiological attack on a metropolitan area in order to provide information that can be used for the establishment of early response measures. Seven radioactive materials were selected as source materials for the evaluation. The results showed that Am-241 is the most dangerous material among the radioactive sources which can be used in a RDD. Other important factors for determining consequences are explosive power and meteorological conditions--especially the intensity of winds. It was revealed from this study that the TEDE decreases with the increase of explosive power and wind intensity. The wind direction and intensity that was used in this study was obtained from analyzing meteorological data for the last 25 years in Korea.

Besides meteorological information, type of urban building has an important effect on exposure to the public. The type of surface contamination is determined by the building material--types and distribution. Won Tae Hwang et al. evaluated the exposure doses of surfaces of Korean urban buildings. The method to calculate the effects of building type was overly sophisticated and time consuming process. It is not practical for radiological accident analysis which requires a fast initial assessment - simple, fast and easy-to-use.

A tool by which the evaluation results can be assessed easily is needed to facilitate the use of the database obtained from this study. Currently, a system that enhances the accessibility of the evaluation results is being developed.

\section{Conclusion}

Scenarios on radiological terror in metropolitan areas were developed; and the consequences of each scenario were evaluated. The data used to create the parameters include: radioactive materials, explosive types and meteorological conditions. Seven radioactive materials were selected for evaluation based on information such as frequently used material in nuclear facilities located in metropolitan areas and accident history. Meteorological conditions were determined after analyzing weather data for the last 25 years. The results show that Am-241 is the most harmful radioactive material to both the public and the environment. The total TEDE value caused by an explosion of one $\mathrm{Ci}$ of Am-241 was higher than that of $100 \mathrm{Ci}$ of Co-60. The TEDE value decreases with the increase in the quantity of explosives used and wind intensity. It is thought that radioactive particles are dispersed over a wider area if the detonation is more powerful and the winds blows faster. However, the concentration of radioactive particle would be diluted if the same amount of radioactive material diffuses over wider area. The consequence data obtained from this study can be used for the establishment of response measures against radiological terror. In order to enhance its effectiveness, more data for evaluation based on reliable scenarios is needed. In addition, further study on developing a framework that will facilitate the accessibility of data should be required.

\section{Acknowledgments}

This study was performed as a part of the 'long term research and development plan for nuclear energy' supported by the Ministry of Education and Science and Technology. 


\section{References}

H. Kelly. (2002). Dirty bombs: Response to a threat. FAS Public interest report. J. fed of Am. sci, 55, 6-10.

H. Rosoff, D. V. Winterfeldt. (2007). A Risk and Economic Analysis of Dirty Bomb Attacks on the Ports of Los Angeles and Long Beach. Risk Anal, 27, 533-546. http://dx.doi.org/10.1111/j.1539-6924.2007.00908.x

H. W. Henry. (2007). Guiding Resource Allocations Based on Terrorism Risk. Risk Anal, 27, 597-606. http://dx.doi.org/10.1111/j.1539-6924.2007.00909.x

J. D. Matthew, S. F. Paul. (2006). An Integrated Physical Dispersion and Behavioral Response Model for Risk Assessment of Radiological Dispersion Device (RDD) Events. Risk Anal, 26, 501-514. http://dx.doi.org/10.1111/j.1539-6924.2006.00742.x

K. G. Andersson, T. Mikkelsen, P. Astrup, et al. (2008). Estimation of health hazards resulting from a radiological terrorist attack in a city. Radiation Protection Dosimetry, 1-11.

Lawrence Livermore National Laboratory. (2004). HOTSPOT: Health Physics Codes for the PC. Hotspot version 2.05, US DOE

M. Durante, L. Manti. (2002). Estimates of radiological risk from a terrorist attack using plutonium. Radiat. Environ. Biophys, 41, 125-130.

RMS. Managing Terrorism Risk in 2004. Newark: CA Risk Management Soultions.

T. O. Tengs, M.E. (1995). Aams. Five hundred lifesaving interventions and their cost effectiveness. Risk Anal. 15, 369-390. http://dx.doi.org/10.1111/j.1539-6924.1995.tb00330.x

Vladimir P. Reshetin. (2005). Estimation of radioactivity levels associated with a $90 \mathrm{Sr}$ dirty bomb event. Atmospheric Environment, 39, 4471-4477. http://dx.doi.org/10.1016/j.atmosenv.2005.03.047

Won Tae Hwang, Eun Han Kim, Hyo Joon Jeong, et al. (2007). A model for radiological dose assessment in the urban environment. Journal of Radiation Protection, 32, 1-8. 
Table 1. Input Parameters for evaluation

\begin{tabular}{|c|c|c|c|}
\hline Radioactive materials & Explosive & Intensity of wind & Stability of Atmosphere \\
\hline Am-241 & & & \\
Co-60 & & & A \\
Cs-137 & $50 \mathrm{lbs}$ & $1.3 \sim 4.5 \mathrm{~m} / \mathrm{sec}$ & (Stable) \\
Ir-192 & $5001 \mathrm{bs}$ & & $\mathrm{F}$ \\
Ni-63 & & & (Unstable) \\
LEU & & & \\
Pu-239 & & & \\
\hline
\end{tabular}

Table 2. Variation of maximum TEDE value with the types of radioactive materials

\begin{tabular}{|c|c|c|}
\hline \multicolumn{2}{|c|}{ Input parameter } & $\begin{array}{c}\text { Maximum TEDE } \\
\text { (Rem) }\end{array}$ \\
\hline Radioactive Material & Quantity(Ci) & 0.702 \\
\hline Am-241 & 1 & 0.544 \\
\hline Co-60 & 100 & 0.125 \\
\hline Cs-137 & 100 & 0.175 \\
\hline Ir-192 & 100 & 0.188 \\
\hline LEU(5\% U-235) & 1000 & 0.010 \\
\hline Ni-63 & 1000 & 0.488 \\
\hline Pu-239 & 1 & \\
\hline
\end{tabular}

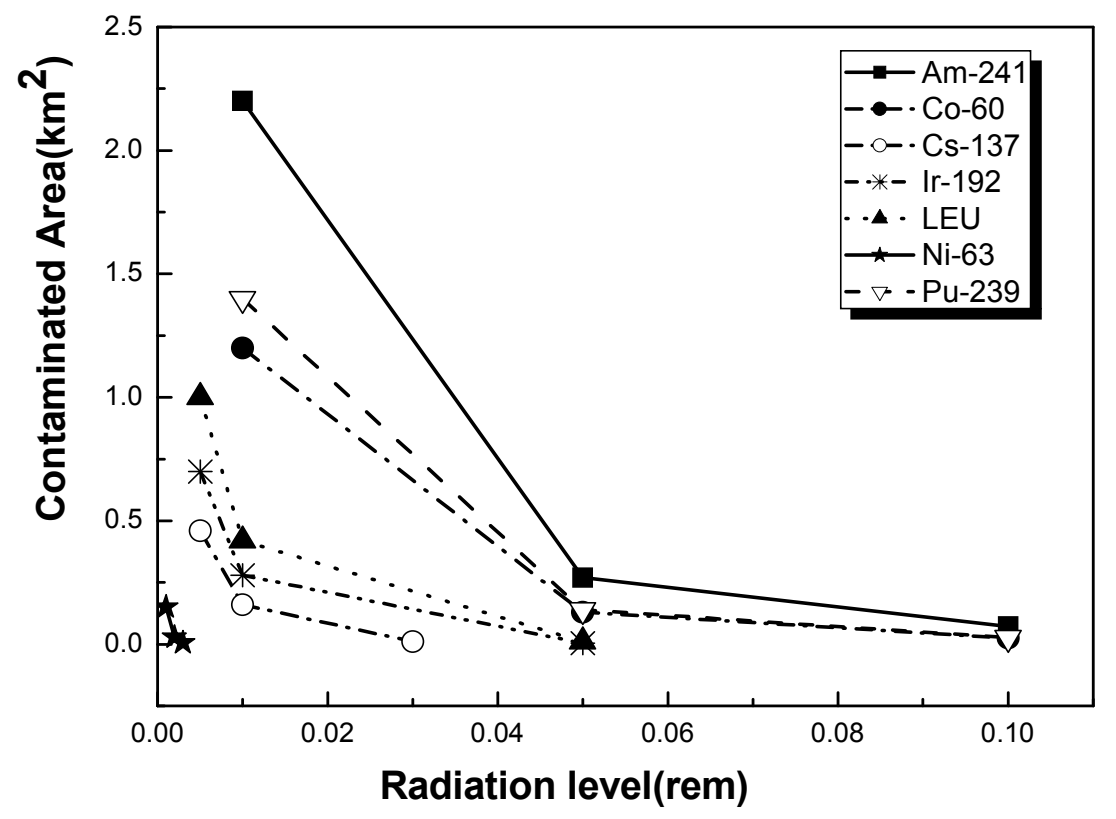

Figure 1. Variation of contaminated area and TEDE value according to source materials 


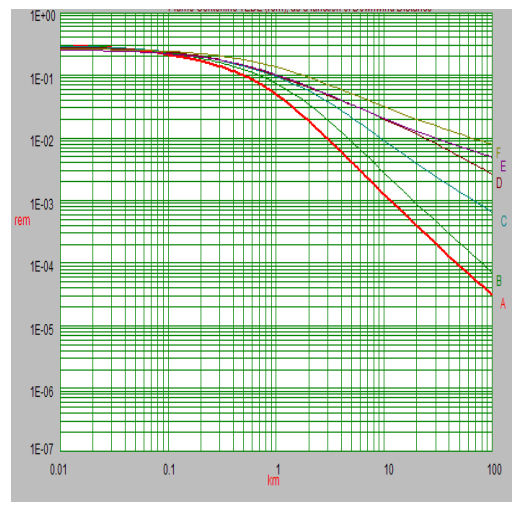

(a) $\mathrm{Am}-241$

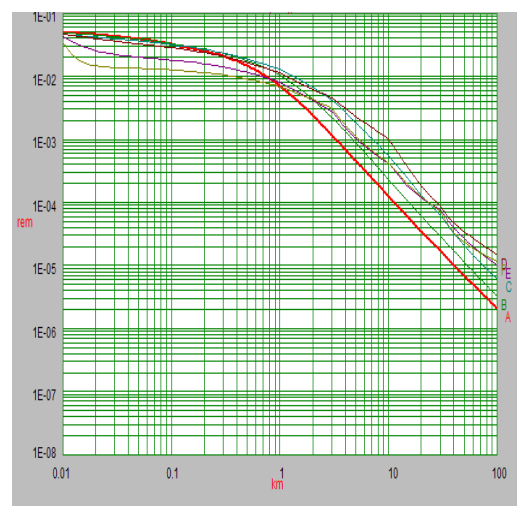

(c) $\mathrm{Cs}-137$

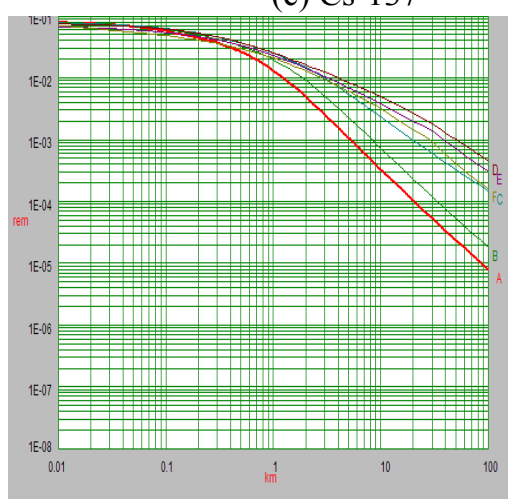

(e) LEU

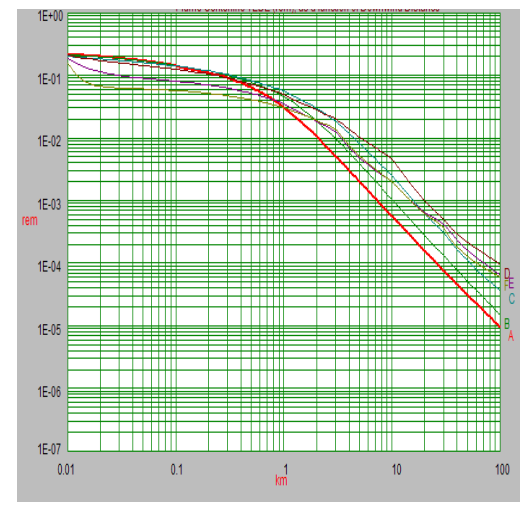

(b) Co-60

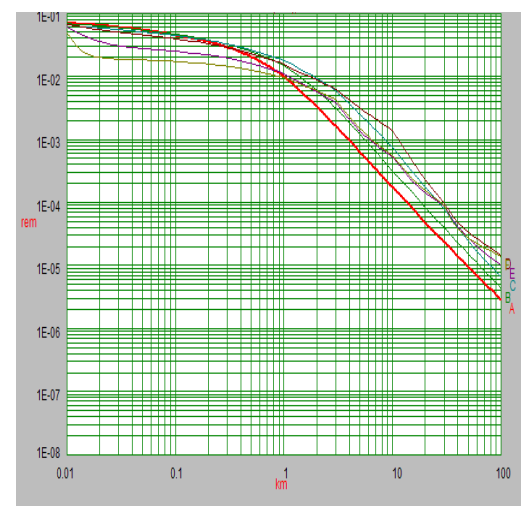

(b) $\operatorname{Ir}-192$

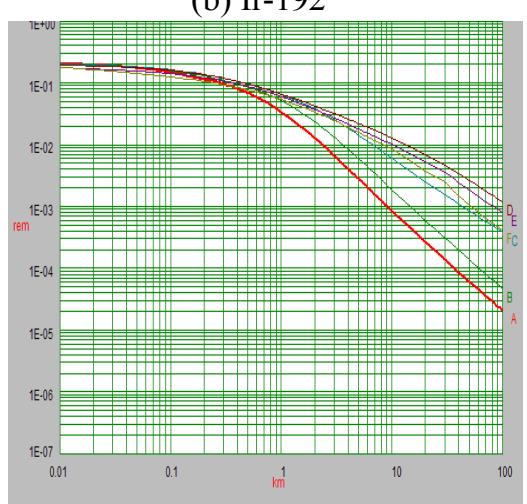

(f) Ni-63

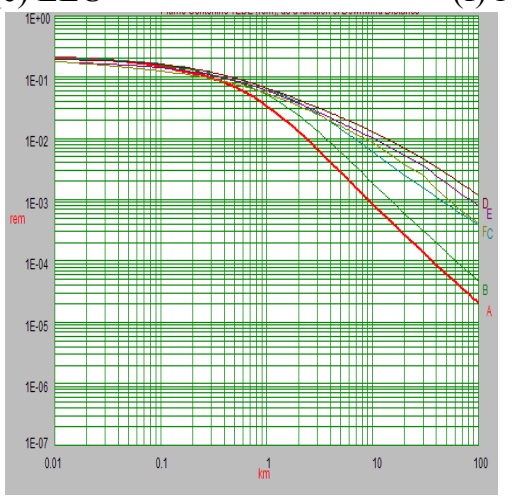

(g) $\mathrm{Pu}-239$

Figure 2. Variation of TEDE value depending on source materials with distance 


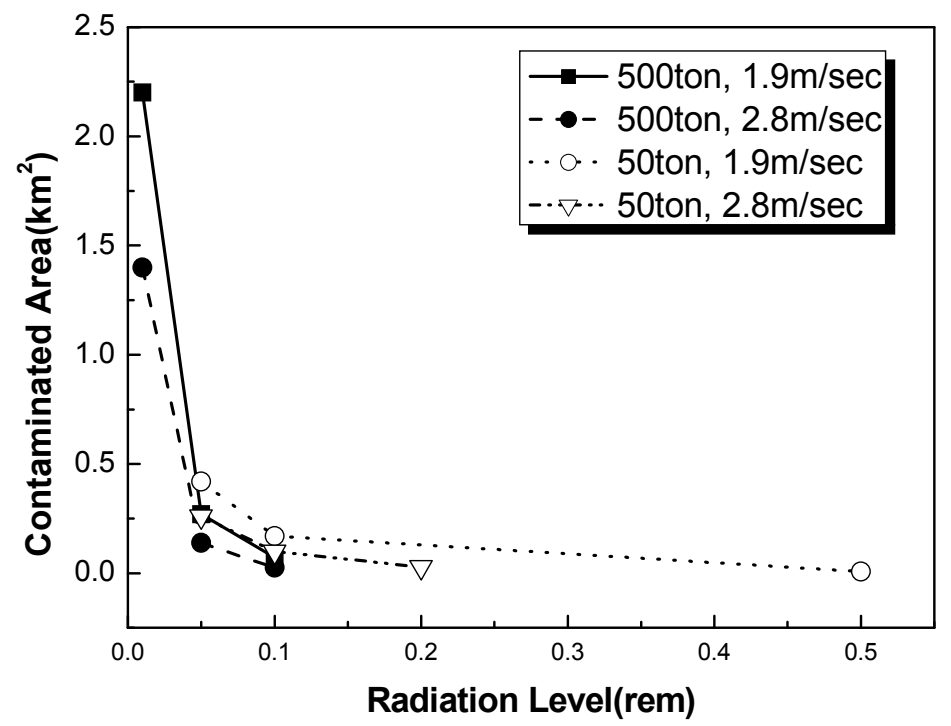

Figure 3. Variation of contaminated area and TEDE value with explosive power and wind intensity (Am-241)

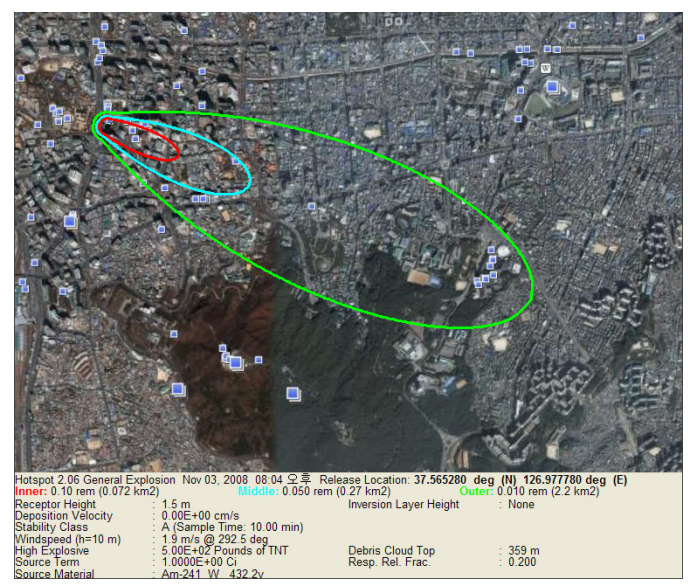

(a)

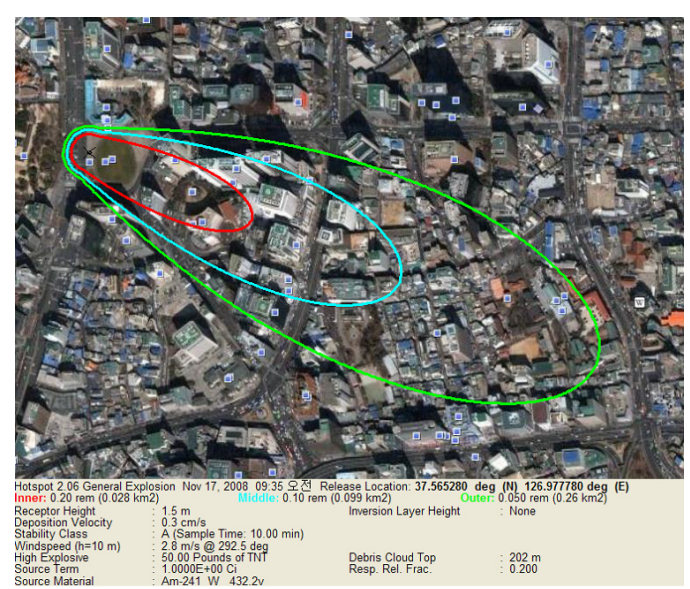

(c)

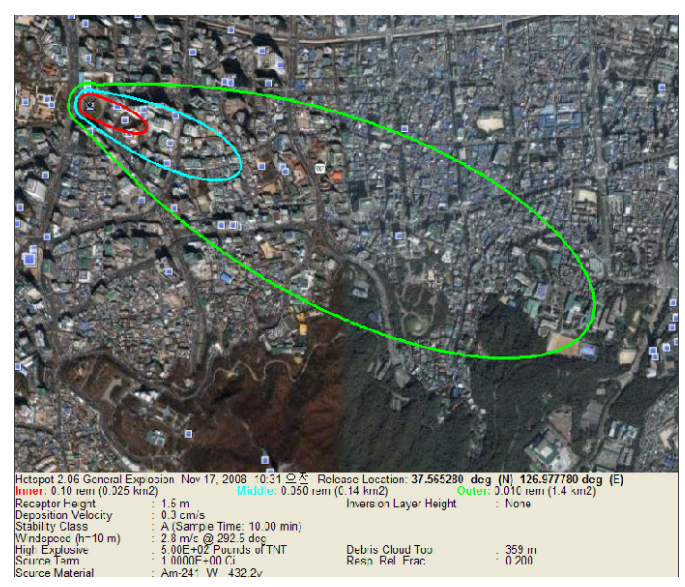

(b)

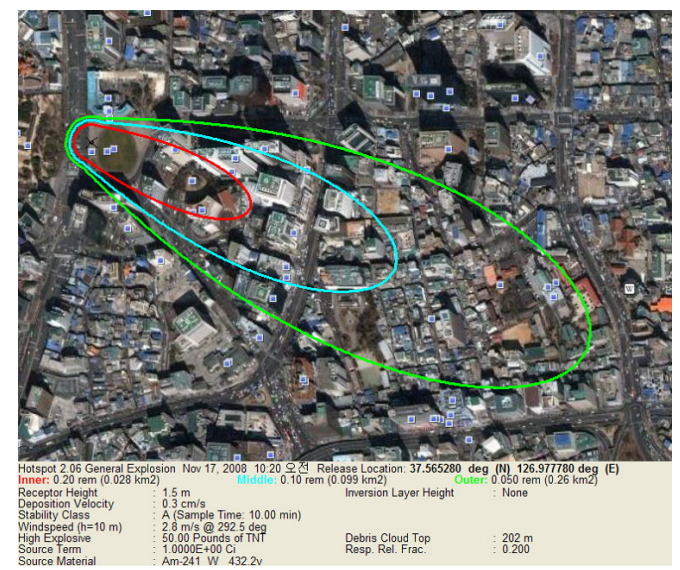

(d)

Figure 4. Variation of plume with explosive power and wind intensity (Am-241)

(a) $500 \mathrm{lbs}, 1.9 \mathrm{~m} / \mathrm{s}$ (b) $500 \mathrm{lbs}, 2.8 \mathrm{~m} / \mathrm{s} \mathrm{(c)} 50 \mathrm{lbs}, 1.9 \mathrm{~m} / \mathrm{s} \mathrm{(d)} 50 \mathrm{lbs}, 2.8 \mathrm{~m} / \mathrm{s}$ 